\title{
Evaluating the Relationship Between Depression and Pain Anxiety with Pain Catastrophizing in Patients with COVID-19
}

\author{
Mahshid Ghasemi (iD) ${ }^{1}$, Faranak Behnaz (iD) ${ }^{2,}{ }^{,}$, Nima Hassanzad (iD) ${ }^{3}$ and Farinaz Taheri (iD) ${ }^{1}$ \\ ${ }^{1}$ Anesthesiology Research Center, Shahid Beheshti University of Medical Sciences, Tehran, Iran \\ ${ }^{2}$ Shohada Tajrish Hospital, Shahid Beheshti University of Medical Sciences, Tehran, Iran \\ ${ }^{3}$ Shahid Beheshti University of Medical Sciences, Tehran, Iran \\ "Corresponding author: Shohada Tajrish Hospital, Shahid Beheshti University of Medical Sciences, Tehran, Iran. Email: faranak.behnaz@gmail.com
}

Received 2021 September 10; Revised 2022 February 05; Accepted 2022 February 08.

\begin{abstract}
Background: This study aimed to investigate the relationship between depression and pain anxiety with pain catastrophizing in patients with coronavirus disease 2019 (COVID-19).

Methods: In this descriptive, correlational study, 180 patients with COVID-19 in Akhtar and Imam Hossein hospitals in Tehran, Iran, were included from March 2019 to April 2020. All participants completed three questionnaires, including the Pain Catastrophizing Scale (PCS), Pain Anxiety Symptoms Scale (PASS), and Beck's Depression Inventory (BDI). The data were analyzed using Pearson correlation coefficient and multivariate regression.

Results: There was a positive and significant relationship between the dimensions of rumination, magnification, and helplessness with total score of pain catastrophizing, as well as moderate to severe dimensions with total pain anxiety and depression in patients with COVID-19.

Conclusions: According to the results of regression analysis, pain anxiety based on pain catastrophizing dimensions was statistically significant, so that rumination, magnification, and helplessness could predict pain anxiety and explain a total of $15.1 \%$ of changes in pain anxiety. Also, depression was statistically significant based on dimensions of pain catastrophizing, so rumination, magnification, and helplessness could predict the patients' depression and explain 13.6\% of depression changes.
\end{abstract}

Keywords: COVID-19, Depression, Pain anxiety, Catastrophizing

\section{Background}

Since December 18 to December 29, 2019, five patients with symptoms of acute respiratory syndrome were hospitalized Akhtar and Imam Hossein hospitals, one of whom died (1). Until January 2, 2020, the results of the coronavirus disease 2019 (COVID-19) test were positive for 41 hospitalized patients, about half of whom had diabetes, hypertension, and cardiovascular diseases (2). On January 30, the World Health Organization (WHO) declared COVID19 pandemic a serious health hazard (3). In February, the virus spread to most parts of the world. In Iran, the first death due to the virus was reported in late February 2017 in Qom (4). WHO declared the outbreak of COVID-19 as a public health emergency of global concern (5). The fastspreading nature of this virus and the lack of knowledge about it have increased the virus-related fears (6). Fatalities due to COVID-19 appear to be concentrated in vulnerable populations, such as the elderly and those with underlying chronic diseases (7).
Catastrophizing is a negative cognitive-emotional process that includes components of magnification, helplessness, and rumination. The degree of catastrophizing is one of the most important predictors of pain treatment outcomes and a key variable in behavioral cognition approaches and fear-avoidance model (8). An individual's attitudes and beliefs, as well as sources and methods of coping with the pain, affect how s/he reports pain (9). Pain catastrophizing has a significant effect on pain experiences, and it is one of the small dimensions of pain adaptation with strong and lasting relationships with pain experience. The most important effect of catastrophizing on chronic pain is that patients with pain receive an assessment of pain that may make them more alert to painful or threatening feelings and fear of experiencing painful feelings in the future (10).

Fathi showed that physical and mental function is further disturbed by the expectation of pain (11). The developed bio-psychosocial view not only addresses pain complaints and the associated physical results but also many 
psychological symptoms that can contribute to the development of chronic pain, the persistence of pain symptoms, and response to medical treatment (8). Studies have shown that psychological variables such as attitudes, anxiety, and depression, instead of biomedical factors, should be considered both physically and psychologically. For most patients, the experience of pain is a disgusting experience. As a result, experiencing pain in the future stimulates fear, anxiety, and adaptation (12). Most studies have found that anxiety affects pain (13). It is known that the level of experienced pain is influenced by several factors, such as depression, stress, anxiety, pain catastrophizing, and insomnia. Patients are informed about the severe postoperative pain that may occur, and this may induce anxiety (14). Studies also suggest pain-related anxiety as one of the most important variables contributing to the development and persistence of pain (15).

Depression and anxiety are strongly related to each other, and often these two disorders are experienced together. Anxiety is one of the most important psychological factors in cardiovascular patients, with a prevalence of up to $50 \%$ in patients with myocardial infarction (16). In this regard, in a study found a correlation between anxiety and depression (17).

\section{Objectives}

In patients with COVID-19, pain catastrophizing is higher due to the unknown nature of disease and different effects it has on different people and ages, which may affect other psychological characteristics such as depression and pain anxiety. Therefore, this study was conducted to investigate the relationship between depression and pain anxiety with pain catastrophizing in patients with COVID19.

\section{Methods}

In this descriptive-correlational study, we included 180 eligible COVID-19 patients hospitalized in Akhtar and Imam Hossein hospitals in Tehran, Iran from March 2019 to April 2020. The inclusion criteria were being in the age range of 25 - 75 years, being a COVID-19 case, and holding at least a high school diploma.

The Ethics Committee of Shahid Beheshti University of Medical Sciences approved the study protocol (code: IR.SBMU.RETECH.REC.1399.1355). Based on the criteria related to observance of the minimum ratio of the number of predictors and samples required for each predictor and at least 10-30 individuals per predictor, considering four predictors (rumination, helplessness, magnifica- tion, and pain catastrophizing) and two criteria (depression and pain anxiety), the sample size was considered as 180 individuals ( 30 for each). After explaining the title and objectives of the study to the patients, they were asked to complete an informed consent form. They were also assured that all patient information would be kept confidential. Then, all participants completed three questionnaires, including Pain Catastrophizing Scale (PCS), Pain Anxiety Symptoms Scale (PASS), and Beck's Depression Inventory (BDI).

\subsection{Pain Catastrophizing Scale}

This questionnaire was developed by Sullivan et al. (18), and it assesses different dimensions of pain and the effect mechanism of catastrophizing. This 13-item scale has three subscales of rumination, magnification, and helplessness, which assess negative pain-related thoughts. The response scale is a 5-point Likert scale, where a lower score indicates less catastrophizing. The total score is obtained from adding responses given to each of 13 items between 0 and 52. In a study by Akbari et al. (19), Cronbach's alpha was reported to be 0.65 for rumination subscale, 0.53 for magnification subscale, 0.81 for helplessness subscale, and 0.84 for the total score of the scale.

\subsection{Pain Anxiety Symptoms Scale}

This scale is a self-report tool for anxiety and painrelated fear reactions developed by McCracken (1992). It assesses symptoms of pain-related anxiety, including three subscales of pain relief related escape and avoidance behavior symptoms, fearful assessment of pain, and painrelated physiological anxiety symptoms (20). The brief form of this scale consists of 20 items developed by McCracken and Dhingra (21), and it is based on the 40-item main scale on pain anxiety symptoms. The range of the brief form scores is between 0 and 100, and the subjects should answer questions in a range from 0 (never) to 5 (always). A total score and three subscale scores are obtained; the total score is generally related to many aspects of patients' performance, and three subscales are used for predicting different aspects of patients' performance. The score ranges are as follows: avoidance $(0-35)$, fearful assessment $(0-40)$, physiological response $(0-25)$, and total score $(0-100)$.

\subsection{Beck's Depression Inventory}

This questionnaire was published by Pour et al. is one of the most common tools for measuring depression. It consists of 21 four-choice questions and has an acceptable validity. The average homogeneity coefficient of was reported to be 0.86 . Internal consistency coefficient was 0.78 , 
and retest reliability was $0.73(22)$. In a study, total reliability of depression using Cronbach's alpha coefficient was reported to be 0.87 (23).

After data collection, mean, standard deviation, and frequency were used to classify and summarize the data. For data analysis, Kolmogorov-Smirnov test was used to investigate normality of data distribution. Pearson correlation coefficient was used to determine two-way correlations between variables, and regression test was used. All analyses were performed at 95\% confidence level using the SPSS software version 22.

\section{Results}

In the present study, 146 (81\%) subjects were female, and 34 (21\%) were male. Also, most of the subjects ( $n=74 ; 41.1 \%$ ) had a high school diploma, and 154 (85.5\%) participants were married. The mean age of the subjects was 48.65 years (standard deviation: 14.5 years). Table 1 shows the descriptive statistics and two-way correlation coefficients between the research variables. The total pain catastrophizing score of patients ( $44.55 \pm 5.45)$ was in the above average range $(P$ $<0.001, t=7.85$ ). In addition, the total scores of pain anxiety and depression were $75.44 \pm 9.45$ and $72.54 \pm 5.46$, respectively. In addition, based on Pearson correlation coefficient, the relationship of pain anxiety and depression with pain catastrophizing was significant and positive. Also, a significant positive relationship was found between rumination, magnification, and helplessness $(\mathrm{P}<0.001)$ subscales with pain anxiety and depression $(\mathrm{P}<0.001)$ (Table 1)

To investigate the capability and power of pain catastrophizing dimensions in explaining the pain anxiety and depression, two multivariate regression models were performed (Table 2).

In the first model, pain anxiety regression model based on the dimensions of pain catastrophizing was statistically significant $\left(\mathrm{R}^{2}=0.151, \mathrm{P}<0.001, \mathrm{~F}=5.331\right)$, so that rumination $(\mathrm{P}<0.001, t=3.933, \beta=0.286)$, magnification $(\mathrm{P}<$ $0.001, t=2.14, \beta=0.34)$, and helplessness $(\mathrm{P}<0.001, t=2.34$, $\beta=0.28$ ) were capable to predict pain anxiety and explain $15.1 \%$ of changes in pain anxiety.

In the second model, the depression regression model based on the dimensions of pain catastrophizing was statistically significant $\left(\mathrm{R}^{2}=0.136, \mathrm{P}<0.001, \mathrm{~F}=4.262\right)$, so that rumination $(\mathrm{P}<0.001, t=3.404, \beta=0.246)$, magnification $(\mathrm{P}<0.001, t=2.11, \beta=0.41)$ and helplessness $(\mathrm{P}<0.001, t=$ $2.68, \beta=0.41)$ were capable to predict the patients' depression and explain $13.6 \%$ of depressive changes.

\section{Discussion}

This study aimed to investigate the relationship between pain depression and anxiety with pain catastrophizing in patients with COVID-19. For this purpose, twoway and multivariate relationships of depression and pain anxiety with pain catastrophizing were investigated using Pearson correlation coefficient and multivariate regression model. The study results showed a positive and significant relationship of dimensions of rumination, magnification, and helplessness with total score of pain catastrophizing, as well as moderate to severe dimensions and total pain anxiety and depression in patients with COVID19. This means that high pain catastrophizing is associated with high pain anxiety and depression in patients with COVID-19. Then, two regression models were used to address the following questions: "Which pain catastrophizing dimensions have a greater effect on explaining the pain anxiety and depression?"

The results showed that the roles of all the three subscales of rumination, helplessness, and magnification were significant in explaining pain anxiety and pain depression. In addition, pain anxiety (15.1\%) and depression (13.6\%) could be explained based on rumination, magnification, and helplessness. Our results regarding the relationship between pain catastrophizing and pain anxiety were consistent with the studies by Granot et al. (24), Barabady et al. (25), Dehghan et al. (26), Tabriz et al. (27), and Margaretten et al. (28). For example, Granot et al. (24) showed that PCS could predict postoperative anxiety levels. Barabady et al. (25) also found that pain was positively associated with catastrophizing. Explaining these results, it can be said that the more severe the patient understands the pain, the higher the patient's anxiety, fear, and worry. The high mortality rate of patients with COVID-19, along with its worldwide prevalence caused these patients to show higher catastrophizing and increased anxiety.

Our results regarding the relationship between pain catastrophizing and depression are consistent with the results of Dehghan et al. (26) and Margaretten et al. (28). Previous evidence supports that the introduction of pretreatment with melatonin or gabapentin reduces pain and disability (29).

Among the research limitations, we can mention the mental and psychological conditions of patients, that affected the response, and using a convenience sampling method. 
Ghasemi M et al.

\begin{tabular}{|c|c|c|c|c|c|c|}
\hline Variables & Mean \pm SD & 1 & 2 & 3 & 4 & 5 \\
\hline Rumination & $12.25 \pm 4.34$ & - & & & & \\
\hline Magnification & $13.64 \pm 2.25$ & $* * 0.75$ & - & & & \\
\hline Helplessness & $19.58 \pm 3.74$ & $* * 0.73$ & ${ }^{* * *} 0.78$ & - & & \\
\hline Pain catastrophizing & $44.55 \pm 5.45$ & $* * 0.76$ & $* * 0.79$ & ${ }^{* * *} 0.78$ & - & \\
\hline Pain anxiety & $75.44 \pm 9.45$ & ${ }^{* * *} 0.35$ & ${ }^{* *} 0.29$ & ${ }^{* *} 0.32$ & ${ }^{* * *} 0.44$ & - \\
\hline Depression & $72.54 \pm 5.46$ & ${ }^{* * *} 0.38$ & ${ }^{* *} 0.34$ & ${ }^{* *} 0.36$ & ${ }^{* * *} 0.28$ & ${ }^{* * *} 0.39$ \\
\hline
\end{tabular}

a ${ }^{*} \mathrm{P}<0.05,{ }^{* *} \mathrm{P}<0.01,{ }^{* * *} \mathrm{P}<0.001$

\begin{tabular}{|c|c|c|c|c|c|c|}
\hline Model-predictors & $\mathbf{F}$ & $\mathbf{R}$ & $\mathbf{R}^{2}$ & B (SE) & $\beta$ & $t$ \\
\hline Model 1: pain anxiety & ${ }^{* * 5} 5.331$ & 0.285 & 0.151 & & & \\
\hline Constant value & & & & $3.284(4.89)$ & & 0.663 \\
\hline Rumination & & & & $0.7(0.177)$ & 0.286 & $* * * 3.993$ \\
\hline Magnification & & & & $0.88(0.41)$ & 0.34 & $* * * 2.14$ \\
\hline Helplessness & & & & $0.42(0.18)$ & 0.28 & ${ }^{* * *} 2.34$ \\
\hline Model 2: depression & ${ }^{* *} 4.262$ & 0.257 & 0.136 & & & \\
\hline Constant value & & & & $8.592(5.869)$ & & 1.464 \\
\hline Rumination & & & & $0.721(0.212)$ & 0.246 & $* * 3.404$ \\
\hline Magnification & & & & $0.94(0.24)$ & 0.41 & ${ }^{* * *} 2.11$ \\
\hline Helplessness & & & & $5.42(1.41)$ & 0.41 & $* * 2.68$ \\
\hline
\end{tabular}

$\mathrm{a}{ }^{*} \mathrm{P}<0.05,{ }^{* *} \mathrm{P}<0.01,{ }^{* * *} \mathrm{P}<0.001$

\section{Footnotes}

Authors' Contribution: Mahshid Ghasemi is the first author (50\% contribution) and designed the study. Nima Hassanzad is the second author (15\% contribution) and conducted the data collection and analysis. Farinaz Taheri is the third author (15\% contribution) and conducted the data collection and analysis. Faranak Behnaz is the corresponding author (30\% contribution) and designed the study.

Conflict of Interests: The authors declare that there are no conflicts of interest.

\section{Ethical Approval: IR.SBMU.RETECH.REC.1399.1355.}

Funding/Support: This research received no specific grant from any funding agency in the public, commercial, or not-for-profit sectors.

Informed Consent: After explaining the title and objectives of the study to patients, they were asked to complete the informed consent form.

\section{References}

1. Ren LL, Wang YM, Wu ZQ, Xiang ZC, Guo L, Xu T, et al. Identifi- cation of a novel coronavirus causing severe pneumonia in human: a descriptive study. Chin Med J (Engl). 2020;133(9):1015-24. doi: 10.1097/CM9.0000000000000722. [PubMed: 32004165]. [PubMed Central: PMC7147275].

2. Huang C, Wang Y, Li X, Ren L, Zhao J, Hu Y, et al. Clinical features of patients infected with 2019 novel coronavirus in Wuhan, China. Lancet. 2020;395(10223):497-506. doi: 10.1016/S0140-6736(20)30183-5. [PubMed: 31986264]. [PubMed Central: PMC7159299].

3. World Health Organization. Novel Coronavirus (2019-nCoV): situation report, 11. Geneva, Switzerland: World Health Organization; 2020.

4. New York Times. Iran Reports Its First 2 Cases of the New Coronavirus. New York Times; 2020.

5. Rahimzadeh P, Faiz HR, Farahmandrad R, Hassanlouei B, Habibi A, Hedayati Emami S, et al. Clinical Features and Prognosis of Invasive Ventilation in Hospitalized Patients with COVID-19: A Retrospective Study. Anesth Pain Med. 2020;10(6). doi: 10.5812/aapm.108773.

6. Ali H, Ismail AA, Abdalwahab A. Mental Stress in Anesthesia and Intensive Care Physicians During COVID-19 Outbreak. Anesth Pain Med. 2020;10(5). doi: 10.5812/aapm.106623.

7. Pergolizzi JJ, Magnusson P, LeQuang JA, Breve F, Paladini A, Rekatsina $\mathrm{M}$, et al. The Current Clinically Relevant Findings on COVID-19 Pandemic. Anesth Pain Med. 2020;10(2). e103819. doi:10.5812/aapm.103819. [PubMed: 32754437]. [PubMed Central: PMC7352949].

8. Higgins C, Smith BH, Matthews K. Comparison of psychiatric comorbidity in treatment-seeking, opioid-dependent patients with versus without chronic pain. Addiction. 2020;115(2):249-58. doi: 10.1111/add.14768. [PubMed: 31386238]. 
9. Cunningham NR, Lynch-Jordan A, Barnett K, Peugh J, Sil S, Goldschneider $\mathrm{K}$, et al. Child pain catastrophizing mediates the relation between parent responses to pain and disability in youth with functional abdominal pain. J Pediatr Gastroenterol Nutr. 2014;59(6):732-8. doi: 10.1097/MPG.0000000000000529. [PubMed: 25121521]. [PubMed Central: PMC4241142].

10. Rahimipour M, Shahgholian N, Yazdani M. Effect of hope therapy on depression, anxiety, and stress among the patients undergoing hemodialysis. Iran J Nurs Midwifery Res. 2015;20(6):694-9. doi: 10.4103/1735-9066.170007. [PubMed: 26793255]. [PubMed Central: PMC4700689].

11. Fathi M, Ariamanesh AS, Joudi M, Joudi M, Sadrossadati F, Izanloo A. Hypnosis as an Approach to Control Pain and Anxiety in Anterior Cruciate Ligament Reconstruction and Meniscal Surgeries: Two Case Presentations. Anesth Pain Med. 2019;9(4). e89277. doi: 10.5812/aapm.89277. [PubMed: 31754607]. [PubMed Central: PMC6825366].

12. Leeuw M, Goossens ME, Linton SJ, Crombez G, Boersma K, Vlaeyen JW. The fear-avoidance model of musculoskeletal pain: current state of scientific evidence. J Behav Med. 2007;30(1):77-94. doi: 10.1007/s10865006-9085-0. [PubMed: 17180640].

13. Irandoost F, Nadi MA, Safary S. The effectiveness of Acceptance and Commitment Therapy (ACT) on the rate of chronic pain and pain catastrophizing in women with chronic low back pain.J Res Psychol Health. 2014.

14. Zuidema WP, Oosterhuis JW, van der Heide SM, de Lange-de Klerk E, van der Steeg AF, van Heurn EL. Correlation of Preoperative State Anxiety and Pain Six Weeks After Surgical Correction of Pectus Excavatum. Anesth Pain Med. 2020;10(1). e98969. doi: 10.5812/aapm.98969. [PubMed: 32337168]. [PubMed Central: PMC7158239].

15. Carleton RN, Abrams MP, Asmundson GJ, Antony MM, McCabe RE. Pain-related anxiety and anxiety sensitivity across anxiety and depressive disorders. J Anxiety Disord. 2009;23(6):791-8. doi: 10.1016/j.janxdis.2009.03.003. [PubMed: 19362446].

16. Januzzi JJ, Stern TA, Pasternak RC, DeSanctis RW. The influence of anxiety and depression on outcomes of patients with coronary artery disease. Arch Intern Med. 2000;160(13):1913-21. doi: 10.1001/archinte.160.13.1913. [PubMed: 10888966].

17. Jaafari Z, Farhadi A, Amin Lari F, Mousavi FS, Moltafet H, Dashti E, et al. Prevalence of Depression in Iranian College Students: A Systematic Review and Meta-analysis. Iran JPsychiatry Behav Sci.2021;15(1). doi: 10.5812/ijpbs.101524.

18. Sullivan MJ, Bishop SR, Pivik J. The Pain Catastrophizing Scale: Development and validation. Psychol Assess. 1995;7(4):524-32. doi:
10.1037/1040-3590.7.4.524.

19. Akbari F, Dehghani M, Khatibi A, Vervoort T. Incorporating Family Function into Chronic Pain Disability: The Role of Catastrophizing. Pain Res Manag. 2016;2016:6838596. doi: 10.1155/2016/6838596. [PubMed: 27445620]. [PubMed Central: PMC4904613].

20. Strahl C, Kleinknecht RA, Dinnel DL. The role of pain anxiety, coping, and pain self-efficacy in rheumatoid arthritis patient functioning. Behav Res Ther. 2000;38(9):863-73. doi: 10.1016/s0005-7967(99)00102-3.

21. McCracken LM, Dhingra L. A short version of the Pain Anxiety Symptoms Scale (PASS-20): preliminary development and validity. Pain Res Manag. 2002;7(1):45-50. doi: 10.1155/2002/517163. [PubMed:16231066].

22. Pour SH, Khairkhah Z, Ghalandari S. The relationship between attachment lifestyle with depression and life expectancy. Indian JEduc Inform Manag. 2013;2(2):573-80.

23. Rahmani F, Hashemniyan $\mathrm{K}$. The effectiveness of cognitive emotion regulation on reduction of depression and anxiety symptoms of adolescences. J Psychol Stud. 2016;12(3):47-65.

24. Granot M, Ferber SG. The roles of pain catastrophizing and anxiety in the prediction of postoperative pain intensity: a prospective study. Clin JPain. 2005;21(5):439-45. doi:10.1097/01.ajp.0000135236.12705.2d. [PubMed: 16093750].

25. Barabady A, Baghdassarians A, Memary E, Yazdani A, Barabady A, Sayadi S. Effect of Benson's Relaxation Technique on Propofol Consumption and Preoperative Anxiety of Patients Undergoing Cataract Surgery. Anesth Pain Med. 2020;10(3). e100703. doi: 10.5812/aapm.100703. [PubMed: 32944558]. [PubMed Central: PMC7472786].

26. Dehghan M, Ahmadi A, Jalili S. A study of pain and anxiety/depression severity on patients with nonspecific chronic low back pain. $J$ Shahrekord Univ Med Sci. 2018;20(3):9-19.

27. Tabriz ER, Mohammadi R, Roshandel GR, Talebi R. Pain Beliefs and Perceptions and Their Relationship with Coping Strategies, Stress, Anxiety, and Depression in Patients with Cancer. Indian J Palliat Care. 2019;25(1):61-5. doi: 10.4103/IJPC.IJPC_137_18. [PubMed: 30820104]. [PubMed Central: PMC6388584].

28. Margaretten M, Julian L, Katz P, Yelin E. Depression in patients with rheumatoid arthritis: description, causes and mechanisms. Int J Clin Rheumtol. 2011;6(6):617-23. doi: 10.2217/IJR.11.6. [PubMed: 22211138]. [PubMed Central: PMC3247620].

29. Javaherforooshzadeh F, Amirpour I, Janatmakan F, Soltanzadeh M. Comparison of Effects of Melatonin and Gabapentin on Post Operative Anxiety and Pain in Lumbar Spine Surgery: A Randomized Clinical Trial. Anesth Pain Med. 2018;8(3). doi: 10.5812/aapm.68763. 